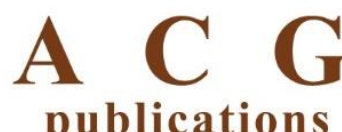

J. Chem. Metrol. 14:1 (2020) 42-51
journal of chemical metrology

publications

\title{
Primary level electrolytic conductivity measurements at National Metrology Institute of Turkey (TUBITAK UME)
}

\author{
Emrah Uysal $\oplus^{1 *}$, Lokman Liv $\odot 1$, Fehmi Fıçıcıoğlu $\oplus^{1}$ and \\ Mehedin Arifoviç ${ }^{2}$ \\ ${ }^{1}$ Electrochemistry Laboratory, TUBITAK UME (National Metrology Institute), Gebze, Türkiye \\ ${ }^{2}$ Voltage Laboratory, TUBITAK UME (National Metrology Institute), Gebze, \\ Türkiye
}

(Received January 13, 2020; Revised February 05, 2020; Accepted February 06, 2020)

\begin{abstract}
TUBITAK UME (National Metrology Institute of Turkey) Electrochemistry Laboratory established the primary level electrolytic conductivity measurement system in 2018. The objective of this work is to prove the accuracy of the established system with an acceptable uncertainty and to disseminate the traceability for electrolytic conductivity measurements. While the accuracy of the system was tested with the certified conductivity solutions, the accuracy of the software developed at TUBITAK UME was also tested. The cell constant was determined as $0.57986 \mathrm{~cm}^{-1}$ at $25^{\circ} \mathrm{C}$ by using certified conductivity solutions. The accuracy of the system was evaluated using the measurement of certified conductivity solutions and it was found that there was no significant difference between the results at 95\% confidence level. Finally, "Evaluation of measurement data - Guide to the expression of uncertainty in measurement" and "EA-4/02 M: 2013 Evaluation of the Uncertainty of Measurement in Calibration" guides were used to form the uncertainty budget for primary level electrolytic conductivity measurements.
\end{abstract}

Keywords: Jones cell; electrolytic conductivity; CRM; traceability. (C) 2020 ACG Publications. All rights reserved.

\section{Introduction}

Electrolytic conductivity (EC) is a measure of its ability to conduct electricity through ions moving in liquids. It is expressed in units such as $\mathrm{S} / \mathrm{m}, \mathrm{mS} / \mathrm{cm}, \mu \mathrm{S} / \mathrm{cm}$. Conductivity measurements in liquids are not specific, all of the ions in a solution contribute to conductivity. However, electrolytic conductivity can be used as a quick and easy method for controlling water quality.

The most important sectors where electrolytic conductivity measurements are applied are described in the following headings:

- Control of water used in pharmaceutical industry,

- Conductivity determination of solutions (fruit juices, soda, soft drinks, etc.),

- Control of the water purity in industrial and scientific laboratories,

\footnotetext{
*Corresponding author E-Mail: emrah.uysal@tubitak.gov.tr
} 
- Control of boiler feed water in industry,

- Determination of salinity of sea waters,

- Determination of total dissolved solid (TDS) in solutions,

- Determination of equilibrium constants (acidity \& solubility constants),

- Conductometric titrations.

Resistance is basically a magnitude that tries to prevent electron movement and is represented by " $R$ ". Also the unit of resistance is expressed in ohm $(\Omega)$. When alternating current is applied to any system, current and voltage passing through the resistor are in the same phase. Reactance is the inertia shown against electron motion. Reactance exists wherever there is an electric field (for a capacitor) that is proportional to the applied voltage or a magnetic field (for a coil) that is proportional to the current. When the alternating current is applied to the pure reactance (the circuit without resistance), there is a $90^{\circ}$ phase shift between the voltage and the current. The reactance is represented by " $\mathrm{X}$ " and its unit is $\mathrm{ohm}(\Omega)$. Impedance consists of resistance and reactance. When the alternating current passes over an impedance, there may be a phase difference between the passing current and the voltage drop between $0^{\circ}$ and $90^{\circ}$. When the phase difference is zero, the impedance is completely resistive, that means there is only a resistance. The ideal resistance contains only a resistance, not a reactance. Similarly, an ideal inductor and an ideal capacitor contain only a reactance, don't contain resistance. However, all circuit elements contain impedance. The impedance may include the resistance and the reactance together [1].

In electrolytic conductivity measurements, the notation of the cell constant is $K_{\text {cell }}$ and the cell constant is formulated as below:

$$
K_{\text {cell }}=\frac{l}{A}\left(\mathrm{~cm}^{-1}\right)
$$

The cell constant depends on the distance $(1 / \mathrm{cm})$ between the two platinum electrodes and the effective surface area $\left(\mathrm{A} / \mathrm{cm}^{2}\right)$ of these electrodes. Resistance measurements are carried out in conductivity measurements. Conductance $(\mathrm{L})$ is the opposite of the resistance.

$$
L=\frac{1}{R} \text { (mho, ohm }{ }^{-1} \text { or } \mathrm{S} \text { (siemens)) }
$$

Accordingly, the conductivity is determined as follows:

$$
k=\frac{K_{\text {cell }}}{R}\left(\mathrm{~S} \cdot \mathrm{cm}^{-1}\right)
$$

The cell used in the primary level electrolytic conductivity measurements is called "Jones Cell". This cell consists of a three-piece glass tube and platinum electrodes at both ends. Jones Cell can be adjusted to different cell constant values due to reduced resistance with a removed middle piece made of quartz called as spacer. The Jones Cell can be used to determine the conductivity of many different solutions. The important thing to consider is to install and disassemble the spacer carefully and ensure the tightness and impermeability [1].

The formulations above are valid for primary level electrolytic conductivity measurements. However, the surface area of the electrode is calculated according to the formula below:

$$
A=3.141593 \times r^{2}
$$

The conductivity of the sample solution is determined by measuring the resistance of the corresponding solution while the cell constant is within a known cell. Two methods can be used to determine the cell constant. The first method is based on measuring the distance between electrodes and the electrode surface area geometrically and accurately and then calculating the ratio of the distance to the area. The second method is based on determination of the cell constant using a certified conductivity 
solution. In the first method, the distance between the electrodes becomes ineffective due to the difference between the resistances obtained while the center tube is present and absent, and the thickness of the center tube and the area of the channel through which the solution passes through the center tube becomes important. According to this statement, the resistance is calculated in line with the formula below:

$$
\overline{\mathrm{R}}_{w}-\overline{\mathrm{R}}_{\mathrm{n}}=\overline{\mathrm{R}}
$$

$\overline{\mathrm{R}}_{\mathrm{w}}=$ Average of resistances in the range $1-8 \mathrm{kHz}$ of the cell without the central section from the LCRmeter

$\overline{\mathrm{R}}_{\mathrm{n}}=$ Average of resistances in the range 1-8 $\mathrm{kHz}$ of the cell with the central section from the LCRmeter

Temperature is another important factor in electrolytic conductivity measurements. Therefore, it is important to measure temperature values very accurately and at high stability $\left( \pm 0.001{ }^{\circ} \mathrm{C}\right)$. According to the effect of these values, the formulation takes its final form as follows:

$$
k=\left(K_{\text {cell }} / \overline{\mathrm{R}}\right) /\left(1+0,0195\left(T-T_{\text {ref }}\right)\right)
$$

$T=$ Measured temperature

$T_{\text {ref }}=$ Reference temperature value $\left(15^{\circ} \mathrm{C}, 25^{\circ} \mathrm{C}, 37^{\circ} \mathrm{C}\right.$ etc. $)$

In the second method, using the equation given in the formula 6, the cell constant can be calculated using the $\mathrm{R}$ value measured by the conductivity value of a certified conductivity solution. In the literature, some studies comparing two methods suggests the use of certified solutions in cell constant determination [2].

All of the laboratories in the country that perform electrolytic conductivity measurements must use reference conductivity solutions to calibrate their systems related to the conductivity measurements and all of these kind of solutions are imported from abroad currently. This application is also a technical and practical necessity in terms of accuracy and traceability of the measurement. Therefore, in order to serve this purpose, production of the certified reference materials (CRMs) for electrolytic conductivity measurements have been planned and nowadays this project has already started. After the production of CRMs, local supply of the certified conductivity solutions will ensure measurement traceability chain through TUBITAK UME and the exchange spent for these solutions will be retained in the country.

Electrolytic conductivity measurements can be incorporated into the International System of Units (SI) as they can be traced to measurements made using a method that fulfils the definition of primary methods of measurement. Due to all these objectives, the establishment of a primary level electrolytic conductivity measurement system, also called as Jones Cell, for measurement of electrolytic conductivity has been carried out.

The raw data is processed and the measurement results are obtained with the help of a software that contains the formulations and makes the calculations accurately.

It was aimed to prove the accuracy of the established system and the software with an acceptable uncertainty level and form the uncertainty budget in detail in the proposed study.

\section{Experimental}

\subsection{Reagents and Solutions}

Potassium chloride $(\mathrm{KCl})$ were obtained from Merck, Emsure level. DFM (Danish Metrology Institute) certified electrolytic conductivity solutions (nominal value of $100 \mathrm{mS} . \mathrm{m}^{-1}$ ) was used for the verification studies. 


\subsection{Auxiliary Equipment}

Milli-Q Integral 10 water purification system was used to produce ultrapure water $(18.2 \mathrm{M} \Omega . \mathrm{cm})$ for preparation of all solutions. Mettler Toledo Seven Excellence Multiparameter conductivity meter was used for EC measurements. HDPE containers and bottles were used for the storage and bottling of the samples, respectively.

\subsection{Primary Level Measurement System}

The system consists of a Fluke Hart Scientific high precision temperature controlled thermostatic bath, a QuadTech 7400 Precision LCR Meter, a KeySight 3458A 81/2 Digit Multimeter, PT100 SRT Temperature Probe, a special software controlled by a computer which was designed at TUBITAK UME and a Jones Cell as a primary level measurement cell. The devices are connected to a PC via GPIB interface and measurements are automatically performed using the software. Figure 1 shows a block diagram and a visual shape of the installed Primary Level Electrolytic Conductivity Measurement System.
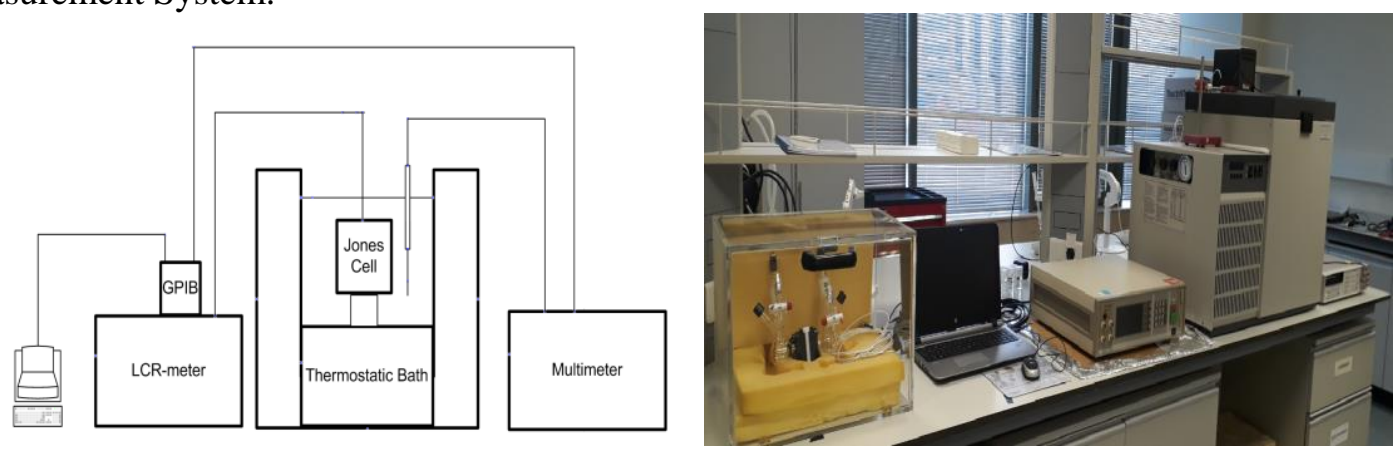

Figure 1. (a) Block diagram of the system, (b) A visual shape of the system

As mentioned above, the cell used for primary level EC measurements is named as "Jones Cell". The construction of this cell consists of part elements that require very precise workmanship. This cell is composed of platinum electrodes at both ends with a three-piece glass tube. The platinum electrodes are in the form of a disc with a diameter of $2 \mathrm{~cm}$. Impermeability between these parts is vitally important for the measurement. The Jones Cell can be set to different cell constant values thanks to the reduced or increased resistance with the removed middle part thus it can be used to determine the conductivity of many solutions with different conductivity levels [3]. The Jones Cell used in the system was produced by an Italian company named DISA where the metrology institutes usually purchase from. Figure 2 and Figure 3 show the Jones Cell and one of the equivalent platinum electrode in the Jones Cell, respectively.

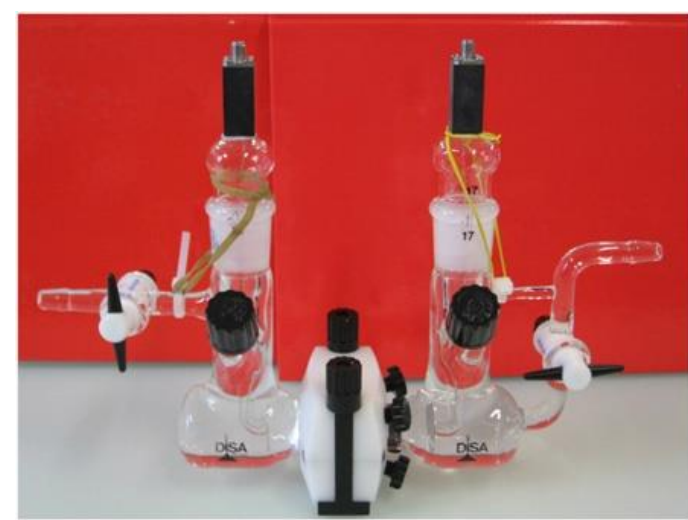

(a)

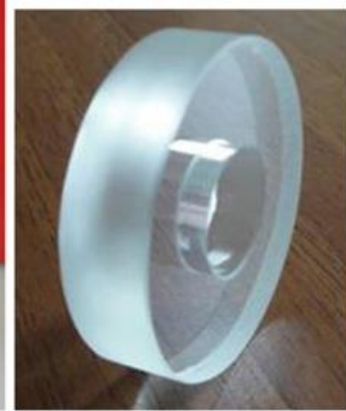

(b)

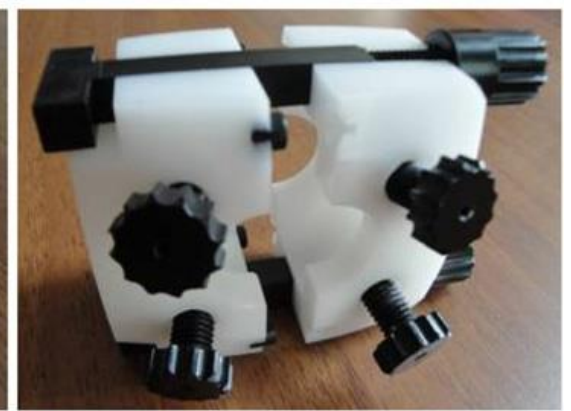

(c)

Figure 2. (a) Jones Cell, (b) intermadiate quartz disc (spacer) and (c) closure system 


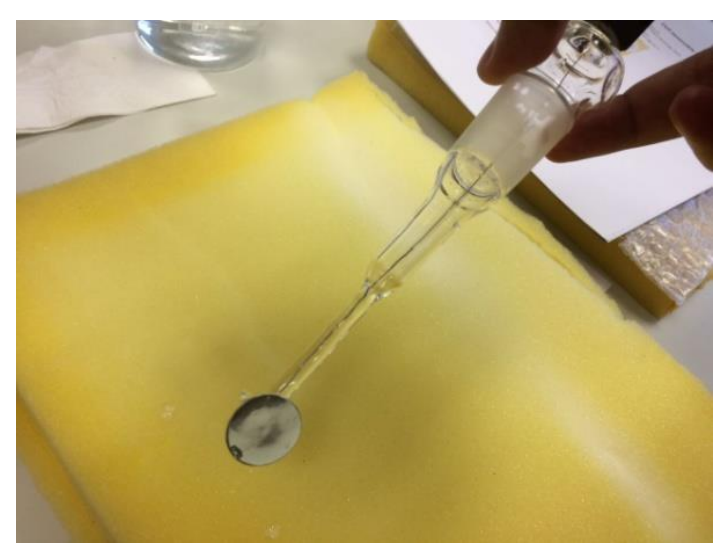

Figure 3. Platinum electrode

\subsection{Measurement Procedure}

A series of measurements were performed by a system with software installed and validated. The purpose of these measurements were to determine the geometrically adjusted cell constant accurately using certified reference conductivity solutions. The other goals of the measurements were to improve measurement repeatability and to reduce measurement uncertainty below the targeted $0.5 \%$ level. According to the literature, the reactance values has been determined as zero in the impedance measurements for values between $0 \mathrm{~Hz}$ and $10 \mathrm{kHz}$. Therefore, it is stated that the primary level conductivity measurements are based on these frequency ranges. After testing in the laboratory for resistance values at increments of $250 \mathrm{~Hz}$ in the $1-8 \mathrm{kHz}$ range, the stability and reproducibility of the resistance values in the frequency range from $3 \mathrm{kHz}$ to $6 \mathrm{kHz}$ were found to be highest.

The Jones Cell should be placed in an oil/water bath with very precise temperature control throughout the entire measurement process in order to make the temperature very stable. A PT-100 SRT temperature probe must be immersed into the bath to monitor and measure the temperature accurately during the measurement [3-5].

For the purpose of determining a cell constant, it was decided that the best way was to use both methods together which were explained in the introduction step above. In the first step, the geometric measurements and calculations of the Jones Cell were realized at Dimensional Laboratory of TUBITAK UME and an approximate cell constant value was set to the actual value. In the second step, several measurements had been performed until the certified value of DFM CRM was equalized with the measurement result obtained by making very sensitive numerical changes on the cell constant used in the software. In order to express the purpose of the procedure for the determination of the cell constant more clearly, when the cell constant was determined, it was based on the comparison between the value of the CRM certificate and the result of the measurement of CRM by the system, while the cell constant value determined geometrically was used as an inception data.

\subsection{System Verification}

The objective of the verification study was to verify the measurements carried out by primary level electrolytic conductivity system. The DFM CRM was used for the verification studies. Temperature value for the experimental conditions were $25.007 \pm 0.002{ }^{\circ} \mathrm{C}(\mathrm{K})$. The results of measurements were also evaluated using t-test analysis in order to demonstrate the accuracy of the measurement results obtained from the system. 


\subsection{Sample Preparation and Measurement}

After the determination of the cell constant and the verification study, a conductivity solution measured by the primary system with an approximately $1408 \mu \mathrm{S} . \mathrm{cm}^{-1}$ conductivity value. In order to prepare this solution, $3.598 \mathrm{~g}$ of Merck, Emsure level potassium chloride $(\mathrm{KCl})$ was weighed in an analytical balance and then diluted to $5 \mathrm{~L}$. It was placed on a shaker for 2 hours for homogenization. Before the measurement, all parts of the Jones Cell were cleaned with deionized water for three times and then placed in a laminar flow cabinet for drying. After this process, approximately $180 \mathrm{~mL}$ of the solution was transferred into the cell. The cell was placed inside the thermostatic bath whose temperature was fixed at $25.000{ }^{\circ} \mathrm{C}$. The temperature of the liquid in the cell was allowed to reach the fixed temperature of the bath for 30 minutes.

\section{Results and Discussion}

\subsection{The Measurement Step}

The cell constant was determined as $0.57986 \mathrm{~cm}^{-1}$ using certified conductivity solutions with a specific conductivity value $\left(999.9 \pm 1.5 \mu \mathrm{S} . \mathrm{cm}^{-1}\right.$ at $\left.25^{\circ} \mathrm{C}\right)$ as a result of the tests explained in 2.4 section.

For the system verification measurements, the electrolytic conductivity measurements of the certified conductivity solutions were repeated for 3 different days. The measurement results for each conductivity value are shown in Table 1 .

Table 1. The measurement results for each conductivity value

\begin{tabular}{cccc}
\hline Replicate & Temperature $\left({ }^{\circ} \mathbf{C}\right)$ & $\mathbf{E C}\left(\boldsymbol{\mu S . c m}{ }^{-1}\right)$ & Certified Value $\left(\boldsymbol{\mu S . c m ^ { - 1 } )}\right.$ \\
\hline 1 & 25.006 & 1001.0 & \\
2 & 25.007 & 1001.2 & $999.9 \pm 1.5$ \\
3 & 25.007 & 1003.9 & \\
\hline
\end{tabular}

The value of $1.5 \mu \mathrm{S} . \mathrm{cm}^{-1}$ presented in the Table 1 with \pm sign correspond to the expanded uncertainty $(\mathrm{k}=2)$. When the sample means of the measurements were compared with the certificated value of the reference solution, the sample means of the measurements were observed within the range of the certificated value according to their uncertainties.

The evaluation was realized using t-test analysis as shown in Eq. 7.

$$
t=\frac{(\overline{\mathrm{s}}-\mu)}{\mathrm{c} / \sqrt{\mathrm{n}}}
$$

where $\overline{\mathrm{x}}$ is sample mean, $\mathrm{s}$ is the sample standard deviation, $\mathrm{n}$ is the number of samples, $\mu$ is the reference value and $v=n-1$ is the degrees of freedom. When $t_{\text {experimental }}$ value is smaller than $t_{\text {critical }}$ value, this means there is no significant difference between the test results at $95 \%$ confidence level and $n-1$ degrees of freedom, then the measurement results are acceptable.

According to the measurement values, the results for t-test are shown in Table 2.

As shown in Table 2, $t_{\text {experimental }} \leq t_{\text {critical }}$ for all results means that there is no significant difference between the measurement results at 95\% confidence level and $n-1$ degrees of freedom. These results provide the method performance criteria for the system.

After the measurements were performed as explained above, the conductivity solution prepared according to the conditions described in section 2.6 was measured by the primary system. The result of the measurement was found as $1405.6 \pm 3.3$. 
Table 2. The results of t-test analysis

\begin{tabular}{cc}
$\bar{x}$ & 1002.1 \\
$\mathrm{~s}$ & 1.6 \\
$n$ & 3 \\
$\mu$ & 999.9 \\
$t_{\text {experimental }}$ & 1.614 \\
$t_{\text {critical }}$ & 2.776 \\
\hline
\end{tabular}

\subsection{Evaluation of the Measurement Uncertainty}

During the evaluation of the measurement results based on the measurements performed at TUBITAK UME, the components of the uncertainties in the uncertainty budget are the standard uncertainties derived from the cell constant, temperature measurements during the measurement, the calibration certificate of PT-100 SRT temperature probe, repeatability and the resistance measurements. The guides of "Evaluation of measurement data - Guide to the expression of uncertainty in measurement" and "EA-4/02 M: 2013 Evaluation of the Uncertainty of Measurement in Calibration" were used in the calculation uncertainty budget and sensitivity coefficients in the uncertainty budget [6]. The uncertainty level between $0.2-0.3 \%$ was achieved by reaching below the targeted uncertainty level. As shown in Figure 4, the greatest contribution to the measurement uncertainty was derived from the cell constant.

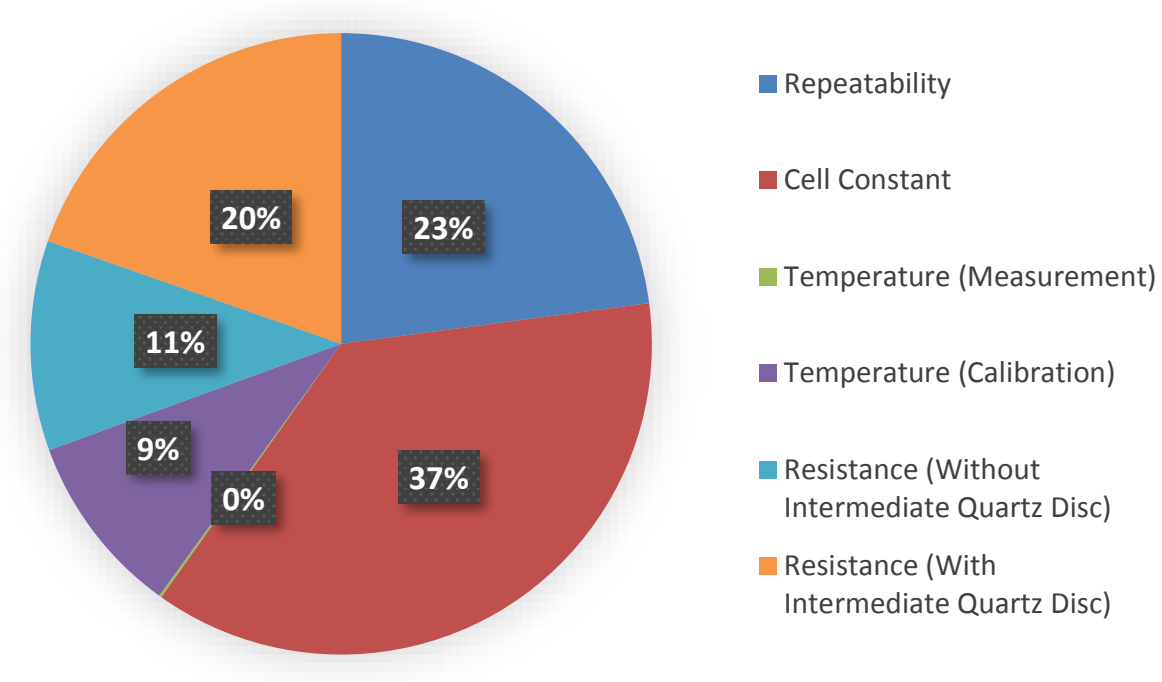

Figure 4. The contributions to the measurement uncertainty

The measurement result and the uncertainty budget of the primary level EC measurement are shown in Table 3.

When taking into considering the results of international comparisons participated by some of National Metrology Institutes (NMIs) that have proven their competence in this field such as PTB (Physikalisch-Technische Bundesanstalt, Germany), NIST (National Institute of Standards and 
Technology, USA), INRIM (Instituto Nazionale di Ricerca Metrologica, Italy), SMU (Slovensky Metrologicky ústav, Slovakia), DFM (Danish Fundamental Metrology, Denmark), GUM (Central Office of Measures, Poland) etc., the expanded uncertainties of measurements were observed between $0.05 \%$ and $0.3 \%$ [7-8]. Therefore it was aimed to reach these uncertainty levels in the measurements to be performed by the established system. If the results from the measurements detailed above were taken into account, it could be seen that the target uncertainty values were obtained.

INRIM is one of the NMIs named above which uses the same Jones Cell design has defined a cell constant as 0.5558 . This value is very close to the value of the cell constant determined in TUBITAK UME which is 0.57986 . This is another proof of achievement for the establishment of recognisable measurement system worldwide.

\subsection{Traceability Chain in Turkey}

The primary level conductivity measurement results are traceable to SI units "Meter", "K" and "Ohm". It should be mentioned that a traceability chain for electrolytic conductivity measurements in Turkey was established with the installation of the primary level measurement system of TUBITAK UME. When the production of primary CRMs for electrolytic conductivity measurements are carried out in the end of 2020, a practical traceability chain will be realized according to the diagram shown in Figure 5.

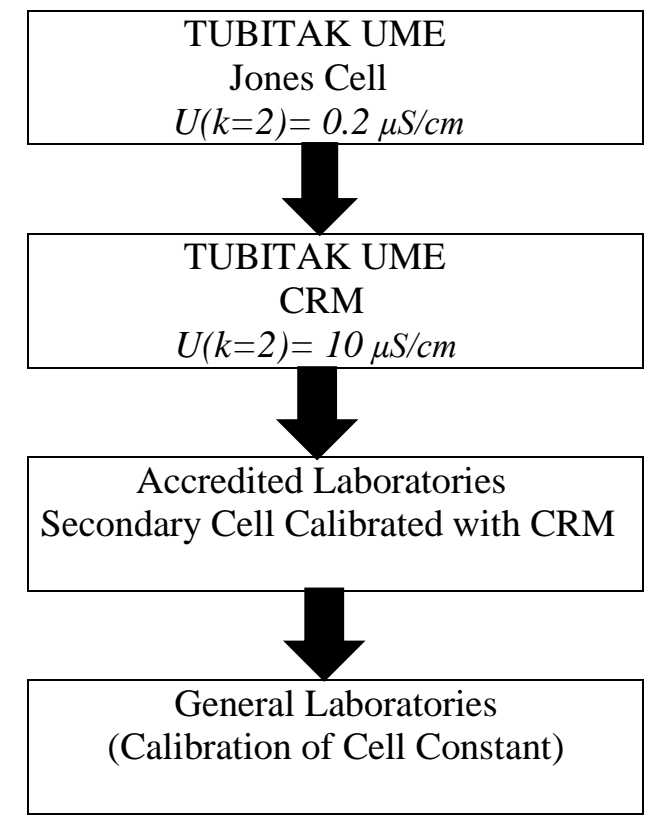

Figure 5. Diagram of the traceability chain in Turkey 
Table 3. Measurement uncertainty budget of primary level EC measurement at TUBITAK UME

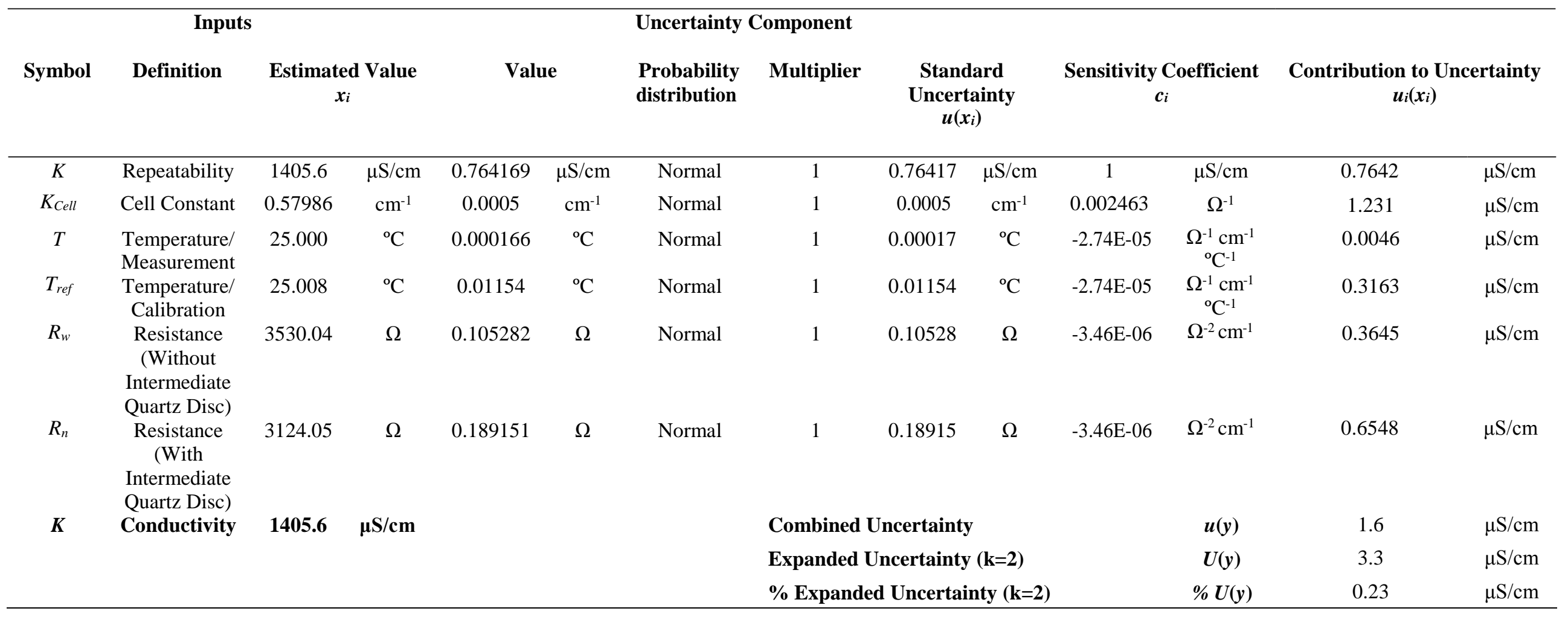




\section{Conclusions}

Primary level electrolytic conductivity system and the software were successfully established and developed at TUBITAK UME. The accuracy of the system was proved by applying student t-test using certified conductivity solutions at $95 \%$ confidence level and the uncertainty evaluations were performed according to "Evaluation of measurement data - Guide to the expression of uncertainty in measurement" and "EA-4/02 M: 2013 Evaluation of the Uncertainty of Measurement in Calibration" guides and the satisfying results were obtained that have expanded uncertainty values below the targeted level as $0.5 \%$. The uncertainty level of the measurements performed in TUBITAK UME Electrochemistry Laboratory by the system shows that it is a significant success to reach the measurement uncertainty levels of the other metrology institutes.

The system has a significant contribution to the related measurements realized in the country and the traceability of the measurements. The system will also provide an opportunity for the production of certified conductivity solutions used for calibration of conductivity meters. The production of the primary level certified conductivity solutions planned to be ended up in the second half of 2020 will close an important deficit in the country in terms of traceability and accuracy of the electrolytic conductivity measurements and fill a gap commercially and economically. The traceability for these measurements will be disseminated through TUBITAK UME subsequent to the production of certified reference solutions. Nevertheless, the laboratory has another step targeted which is to participate of international comparisons will be realized with the established system in order to ensure the compliance with the SI units system for electrolytic conductivity measurements.

\section{ORCID}

Emrah Uysal: $\quad \underline{0000-0001-5783-1874}$

Lokman Liv: $\quad \underline{0000-0001-8008-1012}$

Fehmi Fiçıcıŏlu: 0000-0002-6991-1625

Mehedin Arifoviç: 0000-0002-8616-7287

\section{References}

[1] PTB (2000). Seminar Document: "Electrolytic Conductivity" 146.PTB-Seminar Document.

[2] NIST special publication 260-142: Primary Standards and Standard Reference Materials for Electrolytic Conductivity; 2000.

[3] E. Orru (2013). "Traceability of Electrolytic Conductivity measurements for ultrapure water ", PhD Thesis, Politecnico di Torino, Italy.

[4] J. N. Sørensen (2000). "Establishment of a primary standard for electrolytic conductivity and new reference solutions", Technical University of Denmark and DFM.

[5] IUPAC (2001). Technical Report: Molality based primary standards of electrolytic conductivity.

[6] BIPM (2008). Evaluation of measurement data - Guide to the expression of uncertainty in measurement".

[7] S. Seitz, P. Spitzer and R. J. C. Brown (2010). "CCQM-P111 study on traceable determination of practical salinity and mass fraction of major seawater components", Accred. Qual. Assur. 15,9-17.

[8] L. Vyskočil, M. Máriássy,, A. Reyes, M. Monroy, A.Vospělová, B. Magnusson, R. Pyykkö, E. Kardash, F. B. Gonzaga, I.C.S. Fraga, J.C. Lopes, W.B. Silva Jr, P.P. Borges, W.F.C. Rocha, F. Durbiano, E. Orrù, K.W. Pratt, P.T. Jakobsen, H.D. Jensen, J. Avnskjol, L.A. Konopelko, Y.A. Kustikov, V.I. Suvorov, S. Xiaoping, W. Hai1, S. Seitz, P. Spitzer, V. Gavrilkin, L. Prokopenko, O. Stennik, W. Kozlowski, J. Dumanska-Kulpa, I. Grzybowska, Y.A. Ovchinnikov, Z. N. Szilágyi and J. Fükö (2013). Final report on key comparison CCQMK92: Electrolytic conductivity at $0.05 \mathrm{~S} \mathrm{~m}^{-1}$ and $20 \mathrm{~S} \mathrm{~m}^{-1}$, Metrologia, 50, 0813.

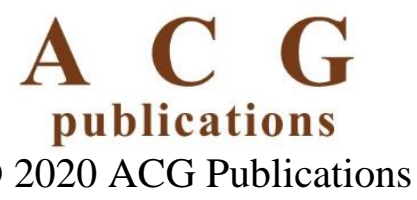

